medgen $2017 \cdot 29: 202-207$

DOI 10.1007/s11825-017-0133-7

Online publiziert: 29. Juni 2017

(c) Der/die Autor(en) 2017. Dieser Artikel ist eine Open-Access-Publikation.

CrossMark

\author{
G. Alex Ochakovski ${ }^{1,2} \cdot$ Karl Ulrich Bartz-Schmidt $^{1}$ M. Dominik Fischer ${ }^{1,2,3}$ \\ ' Universitäts-Augenklinik, Universitätsklinikum Tübingen, Tübingen, Deutschland \\ ${ }^{2}$ Forschungsinstitut für Augenheilkunde, Department für Augenheilkunde, Universitätsklinikum \\ Tübingen, Tübingen, Deutschland \\ ${ }^{3}$ Nuffield Laboratory of Ophthalmology, Nuffield Department of Clinical Neurosciences, University of \\ Oxford, Oxford, Großbritannien
}

\title{
Gentherapie zur Behandlung von Netzhauterkrankungen
}

\section{Einleitung}

Gentherapie am Auge behandelt oder verhindert genetisch bedingte Erkrankungen des visuellen Systems auf der genetischen Ebene. Durch das Einbringen einer therapeutischen Gensequenz in die betroffenen Zellen verspricht dieses innovative Verfahren, bisher unheilbare oder nur unzureichend behandelbare Erbkrankheiten therapieren zu können bzw. deren Progression aufzuhalten.

Die Idee der Gentherapie, deren konzeptioneller Ursprung den 1960er- und 1970er-Jahren entstammt [9], besteht darin, die Ursache der Erbkrankheiten, d. h. die Fehlfunktion des jeweils defekten Gens, durch das Einbringen gesunder Kopien zu beheben. Bei Gentherapie im klassischen Sinne handelt es sich in der Regel um eine Behandlung einer monogenen autosomal-rezessiv vererbten Krankheit, die durch eine Mutation zum Funktionsverlust oder kompletten Ausfall des vom betroffenen Allel abgeleiteten Zielproteins führt (loss of function). Die exogen eingebrachte „Wildtyp“-Gensequenz stellt die Voraussetzung für die Produktion des physiologisch aktiven Proteins wieder her und hemmt damit die Krankheitsentstehung/-progression.

Inzwischen ist gezeigt worden, dass mittels Überexpression der WildtypGensequenz auch autosomal-dominant wirksame gain of function Mutationen behandelbar sind. In bestimmten Fällen erscheint es zusätzlich sinnvoll, z. B. mittels siRNA (short interfering RNA) die Expression des Gens mit gain of function Mutation posttranskriptionell zu limitie- ren [8] und dadurch die Konzentration des toxischen Proteins zu reduzieren.

Eine der vielversprechendsten neuen Richtungen in der Gentherapie ist das sog. „CRISPR-CAS9 Genome Editing“ (CRISPR: clustered regularly interspaced short palindromic repeats; Cas: CRISPRassociated) [23]. Diese Methode basiert auf Bestandteilen eines bakteriellen Abwehrsystems, welches ein gezieltes Editieren bestimmter DNA-Sequenzen im Erbgut nahezu jeder lebendigen Zelle ermöglicht. Mittels einer exogenen RNA-Sonde (sgRNA: single guide RNA) können bestimmte DNA-Sequenzen des Erbguts spezifisch erkannt werden, um sie mithilfe der CAS9-Nukleasen zu entfernen. Nach dem Abbau können die freien DNA-Enden des Genoms mittels NHEJ (non-homologous end joining) oder HDR (homology-directed repair) wieder zusammengefügt werden. Die Methode kann verwendet werden, um z. B. eine Missense-Mutation zu entfernen bzw. diese durch Wildtyp-Sequenz zu ersetzen.

Über die monogenen Erbkrankheiten hinausgehend bietet Gentherapie die Möglichkeit, multifaktoriell bedingte Krankheiten im Rahmen eines allgemeineren Ansatzes zu therapieren. In diesem Zusammenhang wird die allgemeine Widerstandsfähigkeit der betroffenen Zellen gegenüber pathologischen Stressfaktoren (z. B. oxidativer Stress) gezielt und mittels einer gentherapiebedingten Überexpression von Schutzfaktoren verstärkt. Damit könnte das Fortschreiten der zellulären Degeneration unabhängig von der unterliegenden Pathologie unspezifisch behandelt werden. Die Anwendbarkeit dieser Behandlungsstrategien in Gentherapie umfasst ein breites Spektrum von Krankheiten, z. B. Hornhautdystrophien, Glaukom, diabetische Retinopathie, altersbedingte Makuladegeneration (AMD) und hereditäre Netzhautdystrophien. Hier begrenzen wir die Diskussion hauptsächlich auf die klassische retinale Gentherapie, d.h. die Therapie von monogenen, rezessiven Erbkrankheiten der Netzhaut.

\section{Wie erreichen die Gen- sequenzen die Zielzellen am Auge?}

Ein wichtiger Bestandteil der erfolgreichen Gentherapie ist das Einbringen der therapeutischen Gensequenz in die Kerne der Zielzellen. Damit die therapeutischen Gensequenzen die Zellmembran effizient durchqueren können, müssen biomolekulare Vehikel, sog. Vektoren, eingesetzt werden.

Als Vektoren eignen sich prinzipiell unterschiedliche biomolekulare Strukturen. Der ideale Vektor muss aber viele Voraussetzungen erfüllen. Unter anderem soll er die therapeutische Gensequenz in die richtige Zelle mit hoher Selektivität, Effizienz und Sicherheit einschleusen. Dafür soll er idealerweise kaum Immunreaktionen auslösen und darüber hinaus eine ausreichende Kapazität für die betroffenen Gensequenzen bieten.

Dank ausdauernder Erforschung und biotechnologischer Entwicklung sind zahlreiche potenzielle Vektoren für 
Hier steht eine Anzeige.

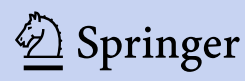


Tab. 1 Die wichtigsten rekombinanten viralen Vektoren im Vergleich

\begin{tabular}{|c|c|c|}
\hline & Lentiviren (LV) & Adenoassoziierte Viren (AAV) \\
\hline Familie & Retroviridae & Parvoviridae \\
\hline Nukleinsäure & RNA & DNA \\
\hline Hülle & Behüllte & Unbehüllte \\
\hline Durchmesser (nm) & $80-100$ & 25 \\
\hline Transgenkapazität (kb) & 10 & 4,8 \\
\hline Integration ins Zellgenom & Willkürlich & Kaum bis keine ${ }^{a}$ \\
\hline Immunogenität im Auge & Deutlich & Äußerst gering \\
\hline $\begin{array}{l}\text { Relevante Erbkrankheiten } \\
\text { (Bsp.) }\end{array}$ & $\begin{array}{l}\text { Morbus Stargardt, Usher-Syndrom 1B, } \\
\text { Makuladystrophien }\end{array}$ & $\begin{array}{l}\text { Achromatopsie, Retinitis pigmentosa, Choroideremie, } \\
\text { Leber'sche kongenitale Amaurose, Makuladystrophien }\end{array}$ \\
\hline Vorteile & $\begin{array}{l}\text { Transduktion mitotischer und postmitotischer } \\
\text { Zellen, größere Kapazität }\end{array}$ & $\begin{array}{l}\text { Nicht humanpathogen, äußerst sicher, } \\
\text { Vielzahl an Serotypen mit unterschiedlichen Gewebe- } \\
\text { affinitäten }\end{array}$ \\
\hline Nachteile & Immunogenität, Unverträglichkeit & Limitierte Transgenkapazität \\
\hline
\end{tabular}

die Gentherapie verfügbar geworden. Grundsätzlich können diese Vehikel in zwei Gruppen eingeteilt werden: die „viralen“ und die „nichtviralen“ Vektoren.

Bei den nichtviralen Vektoren [24] handelt es sich hauptsächlich um mittels Nanotechnologie entwickelte, synthetische biomolekulare Strukturen, die aus polymer- oder lipidbasierten Hüllen oder Kapsiden bestehen. Solche synthetischen Vehikel bieten ein günstiges Immunogenitätsprofil und eine ausreichende Kapazität auch für länge Gensequenzen [26]. Allerdings sind ihre Transduktionseffizienz und Selektivität noch stark verbesserungswürdig, und deshalb ist ihre Einsetzbarkeit in der Gentherapie aktuell relativ begrenzt [2].

Die viralen Vektoren sind jedoch aktuell die am meisten verwendeten Vehikel, mit einem Anteil von ca. $70 \%$ aller gentherapeutischen Versuche [24]. Mit dem Einsatz viraler Vektoren wird auf der Milliarden Jahre langen Evolution der Viren, die die Transduktionseffizienz ihrer Zielzellen perfektioniert hat, aufgebaut. Während des Herstellungsprozesses wird das virale Genom mittels Rekombinationstechnologien durch eine therapeutische Gensequenz ersetzt. Dies führt dazu, dass das Virus seine Replikationsfähigkeit verliert, die Transduktionseffizienz aber erhalten bleibt [22].

Verschiedene Serotypen von Viren haben sich während ihrer Evolution auf die Transduktion bestimmter Zellen spezialisiert. Diese natürlich vorkom- mende Spezialisierung wird während der Auswahlverfahren desjenigen Serotyps des Virus berücksichtigt, der zu den relevanten Zielzellen für die Gentherapie passt und „off-target“ Transduktion minimiert. Die Entwicklung effizienter viraler Vektoren hat sich aber nicht auf natürlich vorkommende Virusvarianten beschränkt. Unterschiedliche Methoden zur biomolekularen Modifikation der viralen Kapside und Hüllen wurden entwickelt und die Vektoren damit noch weiter für spezifische Zwecke optimiert $[6,12]$. Um die Spezifität der Gentherapie weiter zu verbessern, werden spezifische Promotoren verwendet, die idealerweise nur in den Zielzellen exprimiert werden [11].

Die meistverwendeten rekombinanten viralen Vektoren in der Gentherapie am Auge sind Lentiviren und adenoassoziierte Viren (AAV). Beide Vektoren weisen deutliche Unterschiede auf (• Tab. 1). AAVs werden wegen ihrer äußerst geringen Immunogenität, ihrer hohen Transduktionseffizienz und ihrem guten Sicherheitsprofil am häufigsten in der ophthalmologischen Gentherapie benutzt.

Die Blut-Retina-Schranke stellt eine physiologische Barriere nicht nur für Krankheitserreger und Toxine, sondern auch für gentherapeutische Vektoren dar. Um ihre Zielzellen z. B. in der Netzhaut erreichen zu können, müssen die Vehikel in der Regel daher direkt in das Auge injiziert werden. Die Auswahl der Injektionsmethode hat wichtige Auswir- kungen auf die Effizienz, die Sicherheit und den Gesamterfolg der Gentherapie. Die am häufigsten verwendeten Injektionsmethoden in der okulären Gentherapie sind die intravitreale (IVT) und die subretinale (SR) Injektion.

Mit der IVT-Route wird der Vektor in den Glaskörper (Vitreus) injiziert, der den Bulbus zu $90 \%$ ausfüllt und aus Wasser (98 \%), Hyaluronsäure (2 \%) und vereinzelten Kollagenfasern besteht. Diese Injektionsmethode ist relativ sicher und unkompliziert, hat aber gewisse Nachteile für die Gentherapie: Das Volumen des Glaskörpers sowie die Anzahl der exponierten „off-target“ Zellen ist relativ groß. Um das auszugleichen, ist eine höhere Injektionsdosis nötig, die sich aber negativ auf die Immuntoleranz im Auge auswirken kann. Über das Auge hinaus ist mit einer höheren Dosis auch mit einer erhöhten Bioverfügbarkeit außerhalb des Auges zu rechnen, was sich ebenfalls ungünstig auf das Sicherheitsprofil auswirken kann [18]. Wenn die Zielzellen sich in den tiefen Schichten der Netzhaut befinden (z. B. Photorezeptoren oder retinales Pigmentepithel), zeigt schließlich die IVT-Injektion eine geringere Transduktionseffizienz im Vergleich zur SRInjektion.

Bei der SR-Injektion wird die therapeutische Lösung in den potenziellen Raum zwischen der Photorezeptorschicht (PRL) und dem retinalen Pigmentepithel (RPE) gegeben. In manchen Protokollen wird dieser Raum vorab 
mit Kochsalzlösung eröffnet und erst in einem zweiten Schritt die Vektorlösung in den subretinalen Raum injiziert. Innerhalb von ca. $24 \mathrm{~h}$ wird die Lösung fast komplett resorbiert, und damit kehrt die Netzhaut wieder in die Normallage zurück. Die SR-Injektion hat zahlreiche Vorteile: höhere Transduktionseffizienz der RPL- und RPE-Zellen, geringere Anzahl der „off-target“ Zellen, geringere Injektionsvolumen, geringere absolute Menge an Vektorpartikeln, günstigeres Biodistributionsprofil und die Möglichkeit, begrenzte Netzhautareale zu behandeln (z. B. die zentrale Makula). Die SR-Injektion ist aber ein relativ aufwendiger Eingriff, der hochqualifizierter Fachkräfte bedarf. Durch neue Entwicklungen in der chirurgischen Robotik in der Augenheilkunde wird daran gearbeitet, die SR-Injektionen noch sicherer und kontrollierter zu machen.

\section{Das Auge im Visier der aktuellen Gentherapieforschung}

Als doppelt angelegtes, leicht zugängliches und immunprivilegiertes Organ bietet das Auge viele Vorteile für die Erforschung und Anwendung der Gentherapie. Die Sehleistung des Auges kann mittels zahlreicher Modalitäten untersucht und gemessen werden. Das zweite, nicht behandelte Auge wird oft als Kontrollauge benutzt. Die hohe Empfindlichkeit des Auges führt dazu, dass auch die kleinsten Veränderungen in Sehschärfe, Farbwahrnehmung und Gesichtsfeld für den Probanden leicht zu bemerken sind. Elektrophysiologische (ERG) und funktionstomographische (fMRT) Untersuchungsmethoden ermöglichen sogar die Erfassung und Quantifizierung unbewusster Veränderungen des Sehvermögens. Zudem erfasst die Augenheilkunde ein breites Spektrum von Erbkrankheiten mit gut definierten Pathomechanismen.

\section{Leber'sche kongenitale Amaurose}

Die autosomal-rezessiv vererbte Leber'sche kongenitale Amaurose vom genetischen Subtyp 2 (LCA2) ist die erste Erbkrankheit des Auges, für die eine gentherapeutische Behandlung entwickelt wurde. LCA2 liegen loss of function
Mutationen des RPE65-Gens (retinal pigment epithelium-specific $65 \mathrm{kDa}$ protein) zugrunde, das für das gleichnamige Isomerohydrolase-Enzym kodiert. Das RPE65-Enzym sorgt in den RPE-Zellen der Netzhaut für die Regeneration der toxischen Retinolform im Sehzyklus (von all-trans- zu 11-cis-Retinol). Der Funktionsverlust von RPE65 und die nachfolgende Akkumulation des toxischen all-trans-Retinols führen schon in der frühen Kindheit zur progressiven Degeneration des RPEs und der Photorezeptoren.

Die Ergebnisse einer zweijährigen klinischen Follow-up-Studie (NCT007 49957) haben nach SR-Injektion mit rAVV (rAAV2-CB-hRPE65) eine Verbesserung der Sehleistung bei neun von zwölf Probanden gezeigt [20]. Die jüngsten Patienten scheinen von dieser Therapie am meisten profitiert $\mathrm{zu}$ haben. Andere Studien zeigten eine weitere Progression des Zellverlustes auch nach dem Therapiebeginn und eine langsame Verschlechterung der Sehleistung ab drei Jahren nach der Therapie $[5,10]$.

\section{Achromatopsie}

Die Achromatopsie (ACHM) ist eine autosomal-rezessive Erbkrankheit, die eine angeborene komplette Farbblindheit verursacht. Weiterhin äußert sich die ACHM bei Betroffenen in einer stark reduzierteren Sehleistung, starker Lichtscheu und Augenzittern (Nystagmus). Die am meisten verbreiteten Unterformen der Achromatopsie werden von Funktionsverlustmutationen der Gene CNGA3 und CNGB3 (CNG: cyclic nucleotide-gated channel) verursacht. Beide Gene kodieren für unterschiedliche Untereinheiten des gleichen cGMP-gesteuerten Kationenkanals (CNG-Kanal), der sich in den Zapfen-Photorezeptoren befindet [15].

Nach vielversprechenden Ergebnissen der ACHM-Gentherapie mit rAAV-Vektoren im Tierversuch $[3,14]$ haben die ersten interventionellen klinischen Studien für CNGA3 (NCT02610582) und CNGB3 (NCT02599922) ACHM in 2015 und 2016 begonnen. Die Ergebnisse dieser Studien werden voraussichtlich 2017 und 2018 verfügbar sein. Eine weitere medgen $2017 \cdot 29: 202-207$

DOI 10.1007/s11825-017-0133-7

(c) Der/die Autor(en) 2017. Dieser Artikel ist eine Open-Access-Publikation.

\section{G. A. Ochakovski · K. U. Bartz-Schmidt • M. D. Fischer \\ Gentherapie zur Behandlung von Netzhauterkrankungen}

\section{Zusammenfassung}

Eine Reihe von Netzhauterkrankungen hat bekannte genetische Ursachen, die prinzipiell durch Gentherapie behandelt werden können. Diese Übersicht stellt das Prinzip und die Besonderheiten der okulären Gentherapie dar, fasst den aktuellen Stand der Forschung bis hin zur klinischen Anwendung zusammen und gibt einen Ausblick auf aktuelle Entwicklungen der Gentherapie am Auge.

\section{Schlüsselwörter}

Gentherapie · Netzhaut · Monogene

Erbkrankheiten AAV

\section{Gene therapy as a treatment for retinal disease}

\section{Abstract}

A wide spectrum of retinal diseases with well-defined genetic causes can potentially be treated using gene therapy. In this review, we present the basic principles and peculiarities of ocular gene therapy. We discuss the current state of research and transition into clinical application, in addition to recent developments in gene therapy for the eye.

\section{Keywords}

Gene therapy · Retina - Monogenic hereditary disorders · Adeno-associated virus

interventionelle Studie zur CNGA3-assoziierten Achromatopsie (NCT02935517) ist für 2017 geplant.

\section{Choroideremie}

Choroideremie (CHE) ist eine rezessive $\mathrm{X}$-chromosomal bedingte Erbkrankheit, die durch Mutationen des CHM-Gens, das für REP1 (Rab-escort protein 1) kodiert, zur kompletten Atrophie der Aderhaut, RPE und Netzhaut führt. Die ersten Schritte der Krankheitsprogression sind bei CHE-Betroffenen schon in der ersten Lebensdekade zu erkennen. Die 
Atrophie beginnt in der mittleren Peripherie der Netzhaut und schreitet in Richtung Zentrum fort. In der fünften Lebensdekade, vor einer kompletten Erblindung, bleibt nur eine kleine zentrale Restinsel auf der Netzhaut, die nur einen begrenzten Tunnelblick ermöglicht. Die heterozygoten Trägerinnen der Mutation bemerken normalerweise keine Symptome der Krankheit. Allerdings sind in der Regel Veränderungen der Netzhaut bei solchen Patientinnen bei einer genaueren Untersuchung zu sehen [25].

Eine sicherheitsorientierte Phase IStudie (NCT01461213) hat eine signifikante Verbesserung nach einer SRInjektion der rAAV2.REP1 gezeigt [13]. Sechs weitere interventionelle Phase I/II klinische Studien mit rAAV-Vektoren werden seit 2015 durchgeführt, zwei davon (NCT02341807 und NCT02407678) sind in der Patientenrekrutierungsphase. Die ersten Ergebnisse aus Phase IIStudien werden voraussichtlich 2017 verfügbar sein.

\section{Die größten Herausforderungen und Zielsetzungen}

Die wichtigsten Herausforderungen der retinalen Gentherapie sind die Vermeidung sicherheitsrelevanter Nebenwirkungen, wie Entzündung oder Reaktionen des Immunsystems, sowie die dauerhafte Wirksamkeit der Gentherapie.

Da die Gentherapie derzeit auf rekombinanten AAV-Viren basiert, besteht die Möglichkeit, dass die angeborenen und erworbenen antiviralen Immunmechanismen des Körpers auch gegen gentherapeutische Vektoren aktiv werden [16]. Bereits bestehende Antikörper gegen AAV können z. B. die Transduktionseffizienz der rekombinanten AAV negativ beeinflussen. Außerdem kann eine zelluläre Immunantwort behandelte Zielzellen als virusinfiziert erkennen und beseitigen [21].

Das Auge ist eines der wenigen immunprivilegierten Organe im Körper [19]. Für die Gentherapie bedeutet dies, dass das Auge und besonders dessen Netzhaut eine relativ gute immunologische Verträglichkeit für Vektoren haben. Um das Risiko für eine Immunreaktion weiter zu senken, werden kurz vor und nach der Behandlung Kortikosteroide eingesetzt [7].

Bezüglich der Wirksamkeit haben die rAAV (rekombinanten AAV) eine sehr gute Transduktionseffizienz im Tiermodell gezeigt. In unterschiedlichen Tiermodellen zeigten sich oft bei denselben rAAV-Stämmen deutliche Unterschiede in der Transduktionseffizienz der retinalen Zellen. Zum Beispiel wies rAAV5 eine hohe Transduktionseffizienz in RPE-Zellen bei Mäusen und Primaten auf, jedoch nicht bei Ratten und Hunden [17]. Daher sind Versuche in Primaten und am Patienten unabdingbar. Das Potenzial der Gentherapie zur Verbesserung der Sehfunktion wurde in unabhängigen klinischen Versuchen gezeigt $[4,13]$.

Die Dauerhaftigkeit der therapeutischen Wirkung ist insbesondere zu einem relevanten Thema geworden, weil die Ergebnisse von zwei LCA-Studien gezeigt haben, dass die Effekte der Therapie nach ca. drei Jahren langsam nachlassen und weiterer Zellenverlust sowie Abnahme der Sehleistung zu beobachten sind. Die dafür verantwortlichen Mechanismen sind noch nicht geklärt $[1,10]$.

\section{Fazit für die Praxis}

- Neue Fortschritte in der Gentherapie umfassen ein immer breiteres Spektrum von Krankheiten.

- Auch Patienten mit komplexen ophthalmologischen Pathologien wie Glaukom und AMD könnten eines Tages von Gentherapie profitieren.

- Erste Ergebnisse der klinischen Studien zeigten eine signifikante Verbesserung der Sehleistung bei Patienten mit Leber'scher kongenitalen Amaurose und Choroideremie.

- Weitere klinische Studien für Choroideremie sind bereits in der Rekrutierungsphase.

- Fragen zur Dauer der Wirksamkeit von Gentherapie müssen noch geklärt werden.

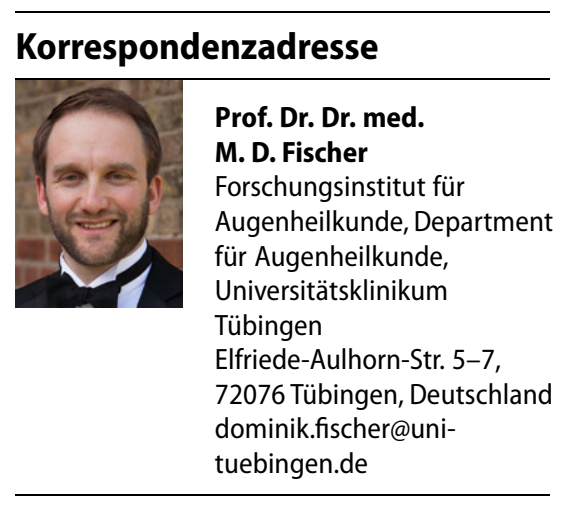

\section{Einhaltung ethischer Richtlinien}

Interessenkonflikt. Diese Arbeit wurde erstellt mit Unterstützung durch die Tistou \& Charlotte Kerstan Stiftung (M. D. Fischer), UK Medical Research Council (MR/K003690/1) (M. D. Fischer), Ewald und Karin Hochbaum Stiftung (M.D. Fischer), Henning Zügel Stiftung (M.D. Fischer) und die Pro Retina Stiftung (M.D. Fischer). M.D. Fischer ist als Berater tätig für NightStaRx Ltd., STZeyetrial und EyeServ GmbH und erhält Honoraria oder Reisekosten von Bayer Vital GmbH und Novartis Pharma GmbH. G.A. Ochakovski und K.U. Bartz-Schmidt geben an, dass kein Interessenkonflikt besteht.

Dieser Beitrag beinhaltet keine von den Autoren durchgeführten Studien an Menschen oder Tieren.

Open Access. Dieser Artikel wird unter der Creative Commons Namensnennung 4.0 International Lizenz (http://creativecommons.org/licenses/by/4.0/deed. de) veröffentlicht, welche die Nutzung, Vervielfältigung, Bearbeitung, Verbreitung und Wiedergabe in jeglichem Medium und Format erlaubt, sofern Sie den/die ursprünglichen Autor(en) und die Quelle ordnungsgemäß nennen, einen Linkzur Creative Commons Lizenz beifügen und angeben, ob Änderungen vorgenommen wurden.

\section{Literatur}

1. Bainbridge JWB, Mehat MS, Sundaram V, Robbie SJ, Barker SE, Ripamonti C, Georgiadis A, Mowat FM, Beattie SG, Gardner PJ, Feathers KL, Luong VA, Yzer S, Balaggan K, Viswanathan A, de Ravel TJL, Casteels I, Holder GE, Tyler N, Fitzke FW, Weleber RG, Nardini M, Moore AT, Thompson DA, Petersen-Jones SM, Michaelides M, van den Born LI, Stockman A, Smith AJ, Rubin G, Ali RR (2015) Longterm effect of gene therapy on Leber's congenital amaurosis. N Engl J Med 372:1887-1897. doi:10. 1056/NEJMoa1414221

2. Brown MD, Schätzlein AG, Uchegbu IF (2001) Gene delivery with synthetic (non viral) carriers. Int J Pharm 229:1-21. doi:10.1016/S03785173(01)00861-4

3. Carvalho LS, Xu J, Pearson RA, Smith AJ, Bainbridge JW, Morris LM, Fliesler SJ, Ding X-Q, Ali RR (2011) Long-term and age-dependent restoration of visual function in a mouse model of CNGB3associated achromatopsia following gene therapy. Hum Mol Genet 20:3161-3175. doi:10.1093/hmg/ ddr218 
4. Cideciyan AV, Hauswirth WW, Aleman TS, Kaushal S, Schwartz SB, Boye SL, Windsor EAM, Conlon TJ, Sumaroka A, Pang J-J, Roman AJ, Byrne BJ, Jacobson SG (2009) Human RPE65 gene therapy for Leber congenital amaurosis: persistence of early visual improvements and safety at 1 year. Hum Gene Ther 20:999-1004. doi:10.1089/hum. 2009.086

5. Cideciyan AV, Jacobson SG, Beltran WA, Sumaroka A, Swider M, Iwabe $S$, Roman AJ, Olivares MB, Schwartz SB, Komáromy AM, Hauswirth WW, Aguirre GD (2013) Human retinal gene therapy for Leber congenital amaurosis shows advancing retinal degeneration despite enduring visual improvement. Proc Natl Acad Sci 110:E517-E525. doi:10.1073/pnas.1218933110

6. Dalkara D, Byrne LC, Klimczak RR, Visel M, Yin L, Merigan WH, Flannery JG, Schaffer DV (2013) In vivo-directed evolution of a new adeno-associated virus for therapeutic outer retinal gene delivery from the vitreous. Sci Transl Med 5:189ra76. doi:10. 1126/scitranslmed.3005708

7. Fischer MD, Wilhelm B, Michalakis S, Zobor D, Kohl $S$, Seeliger M, Zrenner E, Ueffing M, Wissinger B, Biel M, Bartz-Schmidt KU (2016) Successful delivery of rAAV8.CNGA3 in a patient with CNGA3 achromatopsia. Invest Ophthalmol Vis Sci 57:5207

8. de Fougerolles A, Vornlocher H-P, Maraganore J, Lieberman J (2007) Interfering with disease: a progress report on siRNA-based therapeutics. Nat Rev Drug Discov 6:443-453. doi:10.1038/nrd2310

9. Friedmann T, Roblin R (1972) Gene therapy for human genetic disease? Science 175:949-955. doi:10.1126/science.175.4025.949

10. Jacobson SG, Cideciyan AV, Roman AJ, Sumaroka A, Schwartz SB, Heon E, Hauswirth WW (2015) Improvement and decline in vision with gene therapy in childhood blindness. N Engl J Med 372:1920-1926. doi:10.1056/NEJMoa1412965

11. Komáromy $A M$, Alexander JJ, Cooper AE, Chiodo VA, Acland GM, Hauswirth WW, Aguirre GD (2008) Targeting gene expression to cones with human cone opsin promoters in recombinant AAV. Gene Ther 15:1049-1055. doi:10.1038/gt.2008.32

12. Kotterman MA, Schaffer DV (2014) Engineering adeno-associated viruses for clinical gene therapy. Nat Rev Genet 15:445-451. doi:10.1038/nrg3742

13. MacLaren RE, Groppe M, Barnard AR, Cottriall $\mathrm{CL}$, Tolmachova T, Seymour L, Clark KR, During MJ, Cremers FPM, Black GCM, Lotery AJ, Downes SM, Webster AR, Seabra MC (2014) Retinal gene therapy in patients with choroideremia: initial findings from a phase $1 / 2$ clinical trial. Lancet 383:1129-1137. doi:10.1016/S01406736(13)62117-0

14. Michalakis S, Mühlfriedel R, Tanimoto N, Krishnamoorthy V, Koch S, Fischer MD, Becirovic E, Bai $L$, Huber G, Beck SC, Fahl E, Büning H, PaquetDurand F, Zong X, Gollisch T, Biel M, Seeliger MW (2010) Restoration of cone vision in the CNGA3-/mouse model of congenital complete lack of cone photoreceptor function. Mol Ther 18:2057-2063. doi:10.1038/mt.2010.149

15. Michalakis S, Schön C, Becirovic E, Biel M (2017) Gene therapy for achromatopsia. J Gene Med. doi:10.1002/jgm.2944

16. Reichel FF, Peters $T$, Muehlfriedel $R$, Biel $M$, Paquet-Durand F, Ueffing $M$, Wissinger B, BartzSchmidt KU, Fischer MD, Michalakis S (2016) Humoral immune response to subretinal AAV8 in non-human primates. Invest Ophthalmol Vis Sci 57:778-778
17. Rolling F (2004) Recombinant AAV-mediated gene transfer to the retina: gene therapy perspectives. Gene Ther 11:S26-S32. doi:10.1038/sj.gt.3302366

18. Seitz IP, Fischer MD, Michalakis S, Wilhelm B, Kahle $\mathrm{N}$, Zrenner E, Ueffing M, Bartz-Schmidt KU, Biel M, Wissinger B, Peters T (2016) rAAV8 biodistribution and shedding after subretinal injection in nonhuman primates. Invest Ophthalmol Vis Sci 57:4025-4025

19. Streilein JW (2003) Ocular immune privilege: therapeutic opportunities from an experiment of nature. Nat Rev Immunol 3:879-889. doi:10.1038/ nri1224

20. WeleberRG, PennesiME, Wilson DJ, KaushalS, Erke LR, Jensen L, McBride MT, Flotte TR, Humphries M, Calcedo R, Hauswirth WW, Chulay JD, Stout JT (2016) Results at 2 years after gene therapy for RPE65-deficient Leber congenital amaurosis and severe early-childhood-onset retinal dystrophy. Ophthalmology 123:1606-1620. doi:10.1016/j. ophtha.2016.03.003

21. Wylie DE, Sherman LA, Klinman NR (1982) Participation of the major histocompatibility complex in antibody recognition of viral antigens expressed on infected cells.JExpMed 155:403-414

22. Xiao X, Li J, Samulski RJ (1998) Production of high-titer recombinant adeno-associated virus vectors in the absence of helper adenovirus. J Virol 72:2224-2232

23. Xue H-Y, Zhang X, Wang Y, Xiaojie L, Dai W-J, Xu Y (2016) In vivo gene therapy potentials of CRISPRCas9.Gene Ther 23:557-559. doi:10.1038/gt.2016. 25

24. Yin $\mathrm{H}$, Kanasty RL, Eltoukhy AA, Vegas AJ, Dorkin JR, Anderson DG (2014) Non-viral vectors for genebased therapy. Nat Rev Genet 15:541-555. doi:10. 1038/nrg3763

25. Zinkernagel MS, MacLaren RE (2015) Recent advances and future prospects in choroideremia. Clin Ophthalmol 9:2195-2200. doi:10.2147/OPTH. S65732

26. Zulliger R, Conley SM, Naash MI (2015) Non-viral therapeutic approaches to ocular diseases: an overview and future directions. J Control Release 219:471-487.doi:10.1016/j.jconrel.2015.10.007 